\section{Beeinflusst das Interesse für Technik die Einstellung zur Ent- sorgung radioaktiver Abfälle?}

\author{
von Martin Stolle, ITAS
}

Das Bundesministerium für Umwelt, Naturschutz und Reaktorsicherheit (BMU) hat 1999 den „Arbeitskreis Auswahlverfahren Endlagerstandorte“ (AkEnd) ${ }^{1}$ beauftragt, Verfahren und Kriterien zur Auswahl von Endlagerstandorten für radioaktive Abfälle in Deutschland zu entwickeln. Die Arbeit des AkEnd in der letzten Legislaturperiode ist dabei ein erster Schritt im Rahmen eines neuartigen Gesamtkonzepts der Standortfindung.

Ziel und Aufgabe des ITAS-Projekts „Expertenkommunikation im Konfliktfeld der nuklearen Entsorgung" ist die sozialwissenschaftliche Evaluation der öffentlichkeitswirksamen Aktivitäten des AkEnd. Zentrale Forschungsaufgabe des Evaluationsprojektes sind zwei repräsentative Bevölkerungsumfragen, die im Herbst 2001 und im Frühsommer 2002 durchgeführt wurden. Sie haben das Ziel, die Veränderung der Einstellung der Bevölkerung gegenüber Technik, zur Endlagerung von radioaktiven Abfällen sowie zur politischen Partizipation zu messen und die Bekanntheit des AkEnd als Expertenkommission in der breiten Öffentlichkeit festzuhalten.

In diesem Artikel werden einige ausgewählte Ergebnisse der ersten repräsentativen Umfrage des Jahres 2001 dargestellt. Die Befragung wurde so konzipiert, dass damit nach Abschluss beider Studien die Möglichkeit besteht, vergleichende Analysen zwischen den beiden Untersuchungszeitpunkten vorzunehmen.

1 Inhaltliche Schwerpunkte der repräsentativen Bevölkerungsumfrage 2001

Der Fragebogen für die face-to-face-Interviews der repräsentativen Bevölkerungsbefragung gliedert sich in insgesamt drei größere Themenbereiche. Eingeleitet wird mit Fragen zum Informationsverhalten und dessen Intensität, zu Wertorientierungen ${ }^{2}$ und zum Interesse an Politik.

Der erste Themenbereich des Fragebogens bezieht sich auf das Technikinteresse und die
Technikbeurteilung der Befragten, die Umweltfolgen von Technik, Meinungen zur Kernenergie, Kenntnis des Atomausstiegs der Bundesregierung und dessen Bewertung sowie die Bedeutung neuer Technologien für die wirtschaftliche Entwicklung in Deutschland.

Der zweite Bereich konzentriert sich auf das Thema der politischen Partizipation. Es finden sich Fragen dazu, inwieweit die Bevölkerung Vertrauen in verschiedene Institutionen setzt, zur Bewertung der politischen Partizipation im allgemeinen, zu Genehmigungsverfahren technischer Großprojekte und verschiedenen Bedingungen der Partizipation in diesem Zusammenhang sowie zur Errichtung eines Endlagers für radioaktive Abfälle.

Der dritte Themenbereich beinhaltet die speziellen Fragen zur Lagerung von radioaktiven Abfällen und zur Nutzung der Kernenergie. Schwerpunkte sind dabei die Glaubwürdigkeit verschiedener Institutionen und deren Informationen zur Nutzung der Kernenergie, die verschiedenen Entsorgungsalternativen, ein nationales und ein internationales Lagerkonzept, die Kriterien der Endlagerung und die Folgen eines Endlagers für radioaktive Abfälle, die Beurteilung vorhandener Endlager wie Schacht Konrad oder Gorleben und die Rolle der Gemeinde hinsichtlich der möglichen Einrichtung eines Endlagers für radioaktive Abfälle.

Am Ende des Fragebogens befinden sich schließlich Fragen zu sozio-demographischen Aspekten wie z. B. Geschlecht, Alter, Schulbildung, beruflicher Ausbildungsabschluss, Stellung im Beruf, Individual- und Haushaltseinkommen, Parteienpräferenz sowie allgemeine Fragen zum Ablauf des Interviews. Zusätzlich wurden noch Variablen zur Regionalisierung der Umfragedaten erhoben (Bundesland, Postleitzahlen, politische Ortsgröße sowie die Systematik der BIK-Verflechtungsgebiete ${ }^{3}$ ).

Das relativ breit angelegte Themenspektrum des Fragebogens resultiert aus der Anlage des Evaluationsprojekts. In der ersten Welle konnten 3.206 und in der zweiten Welle 2.637 Personen befragt werden. Soweit eine Veränderungsmessung hinsichtlich der Einstellung zur Nutzung der Kernenergie und zur Endlagerung von radioaktiven Abfällen angestrebt wird, lässt sich dies nur mit einem Panel realisieren. 


\section{Empirische Ergebnisse der repräsentati- ven Bevölkerungsumfrage}

Im Mittelpunkt der vorliegenden Darstellung der empirischen Ergebnisse der repräsentativen Bevölkerungsumfrage stehen die Fragen zum Themenbereich der Realisierung von technischen Großprojekten und die damit zusammenhängende Wahrung der Interessen der Anwohner. Weiterhin wird auf die Ergebnisse zur Glaubwürdigkeit der Informationen verschiedener Institutionen zum Thema der Nutzung von Kernenergie eingegangen.

Im dann folgenden Abschnitt wird die Realisierung eines Endlagers für radioaktive Abfälle thematisiert, die Fragen hinsichtlich der weiteren Nutzung der Kernenergie in Deutschland und welche Entsorgungsalternativen für radioaktive Abfälle von den Befragten präferiert werden.

Danach werden die Ergebnisse hinsichtlich verschiedener Kriterien für die Endlagerung radioaktiver Abfälle dargestellt und die von den Befragten geschätzten subjektiven Wahrscheinlichkeiten bezüglich der Folgen der Errichtung eines Endlagers für radioaktive Abfälle dokumentiert.

Im letzten Abschnitt geht es um die Einstellungen zur Nutzung der Kernenergie und die Dringlichkeit des Entsorgungsproblems radioaktiver Abfälle. Insbesondere wird davon ausgegangen, dass die Einstellung zum Entsorgungsproblem radioaktiver Abfälle in Deutschland abhängig ist von einer positiven Einstellung zur Nutzung der Kernenergie bzw. von positiven Bewertungen der Technik im Allgemeinen.

\section{Technische Großprojekte und die Inter- essen der Anwohner}

Inwieweit werden nach Meinung der Befragten die Interessen der Anwohner bei der Errichtung von technischen Großprojekten berücksichtigt? Ein sehr geringer Anteil der Befragten von $16,8 \%$ ist der Auffassung, dass die Interessen der Anwohner überhaupt eine Berücksichtigung finden. Weitere $48,8 \%$ meinen, dass die Interessen nur teilweise berücksichtigt werden und $28,9 \%$ gehen davon aus, dass sie nicht berücksichtigt werden. Schließlich äußern 5,5\% keine dezidierte Meinung zu diesem Thema.
Diejenigen, die davon ausgehen, dass die Interessen der Anwohner Berücksichtigung finden, wurden bei einer folgenden Filterfrage nicht weiter befragt, während alle anderen hinsichtlich der speziellen Umstände einer NichtBeteiligung von Anwohnern bei der Realisierung von technischen Großprojekten eingehender interviewt wurden. Die Ergebnisse zu dieser Frage sind in der Tabelle 1 (nächste Seite), orientiert an der Höhe des jeweiligen Mittelwerts, aufgelistet.

Jeweils mehr als drei Viertel der Befragten bewerten es als äußerst wichtig, dass die Anliegen der Bürger ernster genommen werden, die Information über technische Großprojekte verständlicher sein müssen und dass es auch quantitativ mehr Informationen geben muss. Weiteres wesentliches Element ist in diesem Zusammenhang die gewünschte Beratung durch fachkundige Personen.

Etwas weniger als drei Viertel der Befragten meint, dass eine Beteiligung der Bürger sehr frühzeitig erfolgen muss, dass den Bürgern größere Rechte eingeräumt werden müssen und dass bei Gerichtsverfahren den Bürgern keine hohen Kosten entstehen dürften.

Als ,äußerst unwichtig“ werden alle sieben Themen nur von einer Minderheit von ca. $6 \%$ der Befragten angesehen. Insgesamt betrachtet weisen alle sieben Themenbereiche eine sehr hohe Zustimmung auf, was darauf hinweist, dass diesen Themen generell bei der Errichtung technischer Großprojekte eine zu geringe Beachtung geschenkt wird.

Geht es um Konflikte bei der Einführung von neuen Technologien, so befürworten $72,5 \%$ der Befragten eine Beteiligung der Bürger an neuen Formen der Beteiligung, wie z. B. die Einführung von Runden Tischen zur Beseitigung von Konflikten. 
Tab. 1: Erwartungen zur Berücksichtigung der Anwohnerinteressen bei der Errichtung von technischen Großprojekten (in Prozent) ${ }^{4}$

\begin{tabular}{|c|c|c|c|c|}
\hline & $\begin{array}{c}\text { Äußerst wichtig } \\
- \\
\text { Skalenwerte } \\
(6,7)\end{array}$ & $\begin{array}{c}\text { Mittlere Be- } \\
\text { wertungen- } \\
\text { Skalenwerte } \\
\quad(3,4,5)\end{array}$ & $\begin{array}{c}\text { Äußerst un- } \\
\text { wichtig- } \\
\text { Skalenwerte } \\
(1,2)\end{array}$ & $\begin{array}{l}\text { Mittelwert der } \\
\quad \text { Skala }\end{array}$ \\
\hline $\begin{array}{l}\text { 1. Die Behörden müssen die Anliegen der } \\
\text { Bürger ernster nehmen }\end{array}$ & 82,4 & 12,4 & 5,2 & 6.21 \\
\hline 2. Informationen müssen verständlicher sein & 81,5 & 12,4 & 6,1 & 6.15 \\
\hline 3. Den Bürgern mehr Informationen geben & 77,4 & 17,0 & 5,6 & 6.05 \\
\hline $\begin{array}{l}\text { 4. Der Bürger muss sich an fachkundige } \\
\text { Person wenden können }\end{array}$ & 74,9 & 19,1 & 6,0 & 5.97 \\
\hline $\begin{array}{l}\text { 5. Beteiligung der Bürger muss einem frü- } \\
\text { heren Zeitpunkt erfolgen }\end{array}$ & 72,8 & 21,6 & 5,6 & 5.93 \\
\hline $\begin{array}{l}\text { 6. Den Bürgern müssen größere Rechte } \\
\text { eingeräumt werden }\end{array}$ & 72,4 & 22,2 & 5,4 & 5.93 \\
\hline $\begin{array}{l}\text { 7. Den Bürgern dürfen vor Gericht keine } \\
\text { hohen Gerichtskosten entstehen }\end{array}$ & 72,5 & 21,4 & 6,1 & 5.93 \\
\hline
\end{tabular}

$\mathrm{n}=2.666$ in der Filterfrage, d. h. alle diejenigen, die nicht von einer vollständigen Interessenberücksichtigung in der Vorläuferfrage ausgehen.

In einer weiteren Frage wurden Beispiele verschiedener technischer Großprojekte genannt und die Befragten sollten angeben, inwieweit in diesen Fällen die Relevanz einer Partizipation der Bevölkerung für die Entscheidung einer
Ansiedlung der Anlagen gegeben ist bzw. welche Wichtigkeit dieser zugeschrieben wird (Tab. 2).

Tab. 2: Relevanz der Partizipation der Bevölkerung bei einer Entscheidung über die Ansiedlung technischer Großanlagen $(n=3.206 \text {; in Prozent })^{5}$

\begin{tabular}{|l|c|c|c|c|c|}
\hline & Sehr wichtig & Wichtig & $\begin{array}{c}\text { eher un- } \\
\text { wichtig }\end{array}$ & $\begin{array}{c}\text { Ganz un- } \\
\text { wichtig }\end{array}$ & Mittelwert \\
\hline Endlager für radioaktive Abfälle & 73,3 & 13,7 & 5,4 & 7,7 & 1.47 \\
\hline Müllverbrennungsanlage & 63,8 & 25,0 & 6,9 & 4,3 & 1.52 \\
\hline Atomkraftwerk & 67,5 & 16,1 & 9,5 & 7,0 & 1.56 \\
\hline Chemiefabrik & 61,1 & 24,0 & 9,6 & 5,4 & 1.59 \\
\hline Erdölraffinerie & 48,4 & 35,5 & 11,7 & 4,4 & 1.72 \\
\hline Gentechnische Anlage & 42,0 & 31,0 & 19,7 & 7,3 & 1.92 \\
\hline Wasserkraftwerk & 36,0 & 38,8 & 20,3 & 4,9 & 1.94 \\
\hline Windpark & 31,5 & 36,6 & 25,5 & 6,4 & 2.07 \\
\hline Automobilwerk & 28,8 & 38,0 & 25,8 & 7,4 & 2.12 \\
\hline
\end{tabular}


Eine Beteiligung an der Entscheidung über ein Endlager für radioaktive Abfälle bewerten $87 \%$ der Befragten mit „sehr wichtig“ bzw. „wichtig“. Generell liegen die Bewertungen für alle neun in der Tabelle 2 aufgeführten technischen Anlagen sehr stark im Bereich der $\mathrm{Zu}-$ ordnung zur Wichtigkeit hinsichtlich einer Beteiligung der in der Nähe wohnenden Bevölkerung. Selbst für die Ansiedlung eines Automobilwerkes erachten immer noch $66,8 \%$ der Befragten dies als ,wichtig“ bzw. „sehr wichtig“. Nach Auffassung der Befragten wird somit der Partizipation der Bevölkerung bei der Einrichtung von technischen Großprojekten ein sehr hoher Stellenwert zugeschrieben. Ganz besonders gilt dies in diesem Zusammenhang für die Einrichtung eines Endlagers für radioaktive Abfälle - nicht umsonst steht dieses technische Projekt an der ersten Stelle der Tabelle bei einem Mittelwert der Skala von 1.47.

In einer Faktorenanalyse zeigt sich deutlich, dass die aufgeführten technischen Großanlagen von den Befragten offensichtlich sehr unterschiedlich bewertet werden. Für ein Wasserkraftwerk, einen Windpark und eine Automobilfabrik gelten offenbar andere Maßstäbe einer Müllverbrennungsanlage, einem Atomkraftwerk, einer Chemiefabrik, einer Erdölraffinerie und einer gentechnischen Anlage andere, vermutlich risikoreichere Folgen verbunden werden.

Risikoaspekte bzw. Risikoschätzungen sind von wesentlicher Bedeutung und bei einer Neuansiedlung von technischen Anlagen ist eine Zustimmung der in der Nachbarschaft lebenden Menschen unbedingt erforderlich.

\section{Die Glaubwürdigkeit der Informationen verschiedener Institutionen zur Nutzung der Kernenergie}

Ein weiterer wichtiger Aspekt für die Bewertung technischer Großprojekte sind die Informationen, die die Bevölkerung über diese Anlagen erhält. Neben der reinen Informationsweitergabe spielt jedoch deren Glaubwürdigkeit eine besondere Rolle für die Akzeptanz verschiedener Technologien. Im Rahmen dieser Studie wurde nach der Glaubwürdigkeit von Informationen verschiedener Institutionen hinsichtlich der Nutzung der Kernenergie in Deutschland gefragt (Tab. 3).

Tab. 3: Glaubwürdigkeit der Informationen verschiedener Institutionen zur Nutzung der Kernenergie in Deutschland $(n=3.206 \text {; in Prozent })^{6}$

\begin{tabular}{|l|c|c|c|c}
\hline & $\begin{array}{c}\text { völlig/eher } \\
\text { glaubwürdig } \\
\text { Skalenwerte } \\
(1,2)\end{array}$ & $\begin{array}{c}\text { teils/teils } \\
\text { Skalenwerte } \\
(3)\end{array}$ & $\begin{array}{c}\text { eher/völlig un- } \\
\text { glaubwürdig } \\
\text { Skalenwerte } \\
(4,5)\end{array}$ & Mittelwert \\
\hline Umweltforschungsinstitute & 63,7 & 27,0 & 9,3 & 2.32 \\
\hline Umweltverbände & 56,3 & 32,2 & 11,5 & 2.47 \\
\hline $\begin{array}{l}\text { Bürgerinitiativen gegen Kern- } \\
\text { energie }\end{array}$ & 47,6 & 35,6 & 16,8 & 2.63 \\
\hline Kernforschungszentren & 36,4 & 34,1 & 29,5 & 2.96 \\
\hline Journalisten & 25,7 & 49,6 & 24,7 & 3.02 \\
\hline Bundesregierung & 25,9 & 46,6 & 27,5 & 3.06 \\
\hline Gewerkschaften & 25,7 & 43,8 & 30,5 & 3.11 \\
\hline Kirchen & 26,4 & 32,0 & 41,6 & 3.28 \\
\hline Opposition im Bundestag & 17,8 & 45,5 & 36,7 & 3.29 \\
\hline Atomwirtschaft & 17,4 & 30,1 & 52,5 & 3.54 \\
\hline
\end{tabular}

als für die restlichen, am Anfang der Tabelle aufgeführten Anlagen. Es ist zu vermuten, dass mit einem Endlager für radioaktive Abfälle, mit
Als besonders glaubwürdig werden Informationen zur Nutzung der Kernenergie dann angesehen, wenn sie von Umweltforschungsin 
stituten $(63,7 \%)$, von Umweltverbänden $(56,3 \%)$ und von Bürgerinitiativen gegen die Kernenergie (47,6\%) stammen. Jeweils rund ein Viertel der Befragten hält dagegen die Informationen von Journalisten, der Bundesregierung, der Gewerkschaften und der Kirchen für besonders glaubwürdig. Sehr geringes Vertrauen genießen demgegenüber die KernenergieInformationen der Opposition im Bundestag sowie die der Atomwirtschaft.

Dem „eigentlichen“ Kompetenzträger in Sachen Kernenergie, der Atomindustrie in Deutschland, wird also nur ein sehr geringes Vertrauen von der Bevölkerung entgegengebracht. Die Informationen zur Nutzung der Kernenergie der Atomwirtschaft wird hier von mehr als der Hälfte der Befragten als ,eher oder völlig unglaubwürdig" bewertet.

Der AkEnd agiert als Expertenkommission somit in einem Themenfeld, das durch eine ausgesprochen ungleiche Verteilung von Glaubwürdigkeitszuschreibung bei den Sachinformationen charakterisiert ist. Auf den AkEnd kommt hier eine sehr anspruchsvolle Integrationsaufgabe zu, indem diese Expertenkommission einerseits Umweltforschungsinstitute und andererseits die Atomwirtschaft an der Debatte über die Standortsuche und deren Kriterien beteiligen muss.

\section{Realisierung eines Endlagers für radio- aktive Abfälle}

Im Mittelpunkt dieses Abschnitts stehen spezielle Fragen zur Realisierung eines Endlagers für radioaktive Abfälle.

Welcher Weg der Entsorgung radioaktiver Abfälle wird von den Befragten bevorzugt? Eine Mehrheit der Befragten von 66,9\% geht davon aus, dass ein Endlager für radioaktive Abfälle innerhalb der nächsten 10 Jahre zur Verfügung stehen wird, während weitere $22,9 \%$ davon ausgehen, dass dies erst innerhalb der nächsten 30 Jahre der Fall sein wird. Dass die Endlagerung radioaktiver Abfälle erst durch zukünftige Generationen erfolgen wird, die vermutlich über bessere Technologien verfügen werden, vermuten 10,2 \% der Befragten.

In einer weiteren Frage sollten die Befragten angeben, ob sie eher eine nationale oder eine internationale Lösung der Endlagerung radioaktiver Abfälle bevorzugen. Nur 31,5\% der Befragten strebt eine nationale Lösung an, während die Mehrheit von 55,6 \% eine internationale Lösung bevorzugt. Lediglich von $12,8 \%$ der Befragten wird keine der beiden Möglichkeiten präferiert.

Von denjenigen, die eine nationale Lösung bevorzugen (1011 Befragte), gehen 81,8\% davon aus, dass die Endlagerung radioaktiver Abfälle eine von allen Bundesländern zu lösende Frage darstellt.

Von denjenigen, die eine internationale Lösung bevorzugen (1.784 Befragte), gehen $69,1 \%$ davon aus, dass sich mehrere Länder der Europäischen Gemeinschaft zusammenschließen sollten, um ein gemeinsames Endlager zu errichten. Lediglich 22,4\% bevorzugen ein Land außerhalb der EG.

Wenn es darum geht, dass ein gemeinsames Endlager für radioaktive Abfälle der Europäischen Gemeinschaft auch in Deutschland angesiedelt sein könnte (1232 Befragte, die sich in der Frage davor für ein internationales Endlager ausgesprochen haben), dann sind $38,1 \%$ dafür, $41,7 \%$ dagegen und $20,2 \%$ der Befragten sind unentschieden.

Ein ähnliches Phänomen (,not-in-my-backyard") lässt sich für eine andere Frage feststellen. Wenn es um das Thema geht, ob die Befragten ein Endlager für radioaktive Abfälle in ihrer Region akzeptieren würden, schließt eine deutliche Mehrheit von 80,6\% der Befragten diese Möglichkeit aus.

Geht es schließlich um den Aspekt, ob ein zentrales oder mehrere dezentrale Endlager eingerichtet werden sollten, dann sprechen sich $45,5 \%$ der Befragten für ein zentrales Endlager aus, 33,7 \% bevorzugen mehrere kleine Endlager und ein Fünftel der Befragten hat dazu keine dezidierte Meinung.

\section{Kriterien für die Endlagerung radioakti- ver Abfälle}

Einen sehr breiten Raum im Fragebogen nimmt die Bewertung von Kriterien der Endlagerung radioaktiver Abfälle und die Schätzung subjektiver Wahrscheinlichkeiten möglicher Folgen der Einrichtung eines Endlagers ein.

Zunächst zu den Kriterien für die Endlagerung von radioaktiven Abfälle und deren Bedeutung für die Befragten. Es wurden insgesamt neun Kriterien verwendet. Die Ergebnisse 
zu dieser Frage sind in der Tabelle 4 (siehe nächste Seite) wiederum entsprechend der Höhe der jeweiligen Mittelwerte sortiert.

Auffallend sind durchweg die relativ hohen Prozentsätze, die auf die beiden jeweils höchsten Werte der vorgegebenen Skala entfallen. Angefangen mit den Sicherheitsstandards für ein Endlager, über die Sicherstellung des Schutzes der Umwelt bis zum Schutz der Umwelt gegenüber Radioaktivität vereinigen diese drei Kriterien jeweils mehr als $90 \%$ in der Bewertung der Befragten. Diesen drei Kriterien wird von den Befragten im Vergleich zu den anderen somit eine besonders hohe Bedeutung zugeschrieben.

$87,6 \%$ der Befragten messen auch der Berücksichtigung der Interessen der Anwohner eine hohe Bedeutung zu. Ein Endlager sollte in einer Gegend liegen, in der nur wenige Menschen leben, meinen $86,7 \%$, und dass die Rückholbarkeit der radioaktiven Abfälle gewährleistet sein muss, davon gehen $81,9 \%$ der Befragten aus. Dieses letzte Kriterium steht im Übrigen in einem deutlichen Gegensatz zum AkEnd, der in seinen Kriterien von einer NichtRückholbarkeit der radioaktiven Abfälle ausgeht.

$\mathrm{Ob}$ die radioaktiven Abfälle in einem Bergwerk untergebracht werden und vom umliegenden Gestein eingeschlossen werden sollen, dem messen 77,5\% der Befragten eine hohe oder sehr hohe Bedeutung zu.

Eine eher mittlere Bedeutung wird dagegen den letzten beiden Kriterien in der Tabelle zugemessen. Dazu gehört die Freiwilligkeit der Region für die Ansiedlung eines Endlagers für radioaktive Abfälle und dass sich die ErschlieBungs- und Baukosten in einem vertretbaren Rahmen bleiben müssen.

Vergleicht man die Mittelwerte in der Tabelle 4, so zeigt sich, dass bei der verwendeten siebenstufigen Skala die ersten sieben Mittelwerte in einem Intervall von 6,15 bis 6,73 liegen. Diese Verteilung weist darauf hin, dass die Mehrheit der Befragten allen sieben Kriterien eine sehr hohe Bedeutung zuschreiben und nur eine Minderheit zu Bewertungen mit einer sehr geringen Bedeutung neigen.

Nun zu den Folgen, die mit der Einrichtung eines Endlagers für radioaktive Abfälle verbunden sind. Die Befragten sollten subjektive Wahrscheinlichkeiten schätzen, die sie in Zukunft mit verschiedenen Konsequenzen der
Einrichtung eines Endlagers für radioaktive Abfälle verbinden.

Betrachtet man für diese acht subjektiven Wahrscheinlichkeitsschätzungen in der Tabelle 5 (siehe nächste Seite) den jeweiligen Mittelwert, so zeigt sich, dass für sechs dieser Konsequenzen der Wert höher als .5 ist. Geht man davon aus, dass das Eintreten einer Konsequenz mit dem Wert 1.0 als sicher angesehen werden kann, so besitzen eben diese ersten sechs Konsequenzen einen relativ hohen subjektiven Wahrscheinlichkeitswert.

Einen im Vergleich mit den anderen sehr hohen subjektiven Wahrscheinlichkeitswert besitzen die ersten drei Konsequenzen. Die Befragten gehen insbesondere davon aus, dass die Endlagerregion durch Transporte und Sicherheitsmaßnahmen erheblich beeinträchtigt wird (Mittelwert: .68), dass durch ein Endlager für radioaktive Abfälle die Umwelt einer erheblichen radioaktiven Belastung ausgesetzt wird (Mittelwert: .62) und schließlich, dass die Radioaktivität, die von einem Endlager ausgeht, die Gesundheit der Bevölkerung gefährdet (Mittelwert: .61).

Etwas geringer fallen die Mittelwerte für die folgenden drei Konsequenzen aus: Durch die Einrichtung eines Endlagers entstehen neue Arbeitsplätze (Mittelwert: .56), ein Endlager verhindert weitere Zwischenlager an den Kernkraftwerksstandorten (Mittelwert: .55) und durch den Bau eines Endlagers wird die Entsorgung radioaktiver Abfälle gelöst (Mittelwert: .54).

Lediglich für die beiden Konsequenzen der Verringerung der Transporte mit CastorBehältern und hinsichtlich der Vermeidung schwerer Auseinandersetzungen wie in Gorleben werden geringere Mittelwerte ausgewiesen. Insgesamt betrachtet wird somit das Eintreten dieser Konsequenzen eher nicht erwartet. 
Tab. 4: Die Bedeutung verschiedener Kriterien für die Endlagerung von radioaktiven Abfällen in Deutschland (n=3.206; in Prozent) ${ }^{7}$

\begin{tabular}{|l|c|c|c|c|}
\hline & $\begin{array}{c}\text { sehr hohe } \\
\text { Bedeutung } \\
\text { Skalenwerte } \\
(6,7)\end{array}$ & $\begin{array}{c}\text { mittlere } \\
\text { Bedeutung } \\
\text { Skalenwerte } \\
(3,4,5)\end{array}$ & $\begin{array}{c}\text { sehr geringe } \\
\text { Bedeutung } \\
\text { Skalenwerte } \\
(1,2)\end{array}$ & Mittelwert \\
\hline $\begin{array}{l}\text { Die Sicherheitsstandards für ein Endlager mit } \\
\text { radioaktiven Abfällen müssen besonders streng } \\
\text { sein }\end{array}$ & 93,9 & 5,4 & 0,7 & 6.73 \\
\hline $\begin{array}{l}\text { Der Schutz der Umwelt muss ausreichend sicher- } \\
\text { gestellt werden }\end{array}$ & 93,5 & 5,8 & 0,7 & 6.70 \\
\hline $\begin{array}{l}\text { Eine Belastung der Umwelt durch Radioaktivität } \\
\text { muss ausgeschlossen werden }\end{array}$ & 92,6 & 6,4 & 1,0 & 6.68 \\
\hline $\begin{array}{l}\text { Die Interessen der Anwohner müssen umfassend } \\
\text { berücksichtigt werden }\end{array}$ & 87,6 & 11,5 & 0,9 & 6.50 \\
\hline $\begin{array}{l}\text { Ein Endlager für radioaktive Abfälle muss in der } \\
\text { Gegend liegen, in der wenige Menschen leben }\end{array}$ & 86,7 & 12,2 & 1,1 & 6.49 \\
\hline $\begin{array}{l}\text { Die Rückholbarkeit der radioaktiven Abfälle muss } \\
\text { gewährleistet sein, um Fehler nachträglich korri- } \\
\text { gieren zu können }\end{array}$ & 81,9 & 16,1 & 2,0 & 6.29 \\
\hline $\begin{array}{l}\text { Die radioaktiven Abfälle müssen in einem Berg- } \\
\text { werk und durch das umliegende Gestein sicher } \\
\text { eingeschlossen werden }\end{array}$ & 77,5 & 19,3 & 3,2 & 6.15 \\
\hline $\begin{array}{l}\text { Die Ansiedlung eines Endlagers für radioaktive } \\
\text { Abfälle muss auf der Freiwilligkeit der Region } \\
\text { beruhen }\end{array}$ & 69,1 & 26,5 & 4,4 & 5.87 \\
\hline $\begin{array}{l}\text { Die Erschließungs- und Baukosten müssen in } \\
\text { einem vertretbaren Rahmen bleiben }\end{array}$ & 56,6 & 30,1 & 13,3 & 5.26 \\
\hline
\end{tabular}

Tab. 5: Subjektive Wahrscheinlichkeitsschätzungen für das Eintreten möglicher Folgen der Endlagerung radioaktiver Abfälle in Deutschland ( $n=3206$; in Prozent) ${ }^{8}$

\begin{tabular}{|c|c|c|c|c|}
\hline Für wie wahrscheinlich halten Sie es, dass ... & $\begin{array}{l}\text { Sehr wahr- } \\
\text { scheinlich/ } \\
\text { wahrscheinlich }\end{array}$ & Weder/noch & $\begin{array}{l}\text { sehr unwahr- } \\
\text { scheinlich/ } \\
\text { unwahrschein- } \\
\text { lich }\end{array}$ & Mittelwert \\
\hline $\begin{array}{l}\text {...die Region, in der sich das Endlager befindet, } \\
\text { durch die Begleitumstände, wie Transporte und } \\
\text { Sicherheitsmaßnahmen, erheblich beeinträchtigt } \\
\text { wird? }\end{array}$ & 68,4 & 20,1 & 11,5 & .68 \\
\hline $\begin{array}{l}\text {...durch ein Endlager für radioaktive Abfälle die } \\
\text { Umwelt einer erheblichen radioaktiven Belastung } \\
\text { ausgesetzt wird? }\end{array}$ & 56,7 & 21,6 & 21,7 & .62 \\
\hline $\begin{array}{l}\text {...die Radioaktivität, die von einem Endlager } \\
\text { ausgeht, die Gesundheit der Bevölkerung gefähr- } \\
\text { det? }\end{array}$ & 54,7 & 21,1 & 24,2 & .61 \\
\hline $\begin{array}{l}\text {...durch ein Endlager für radioaktive Abfälle neue } \\
\text { Arbeitsplätze entstehen? }\end{array}$ & 49,3 & 24,6 & 26,1 & .56 \\
\hline $\begin{array}{l}\text {...ein Endlager für radioaktive Abfälle in } \\
\text { Deutschland den Bau weiterer Zwischenlager in } \\
\text { den Kernkraftwerken verhindert? }\end{array}$ & 45,5 & 25,1 & 29,4 & .55 \\
\hline $\begin{array}{l}\text {...die Entsorgung radioaktiver Abfälle durch den } \\
\text { Bau eines Endlagers gelöst wird? }\end{array}$ & 46,8 & 20,6 & 32,6 & .54 \\
\hline $\begin{array}{l}\text {...die Anzahl der Transporte mit Castor-Behältern } \\
\text { verringert wird? }\end{array}$ & 36,0 & 24,1 & 39,9 & .48 \\
\hline $\begin{array}{l}\text {...schwere Auseinandersetzungen wie in Gorleben } \\
\text { vermieden werden? }\end{array}$ & 26,5 & 25,1 & 48,4 & .42 \\
\hline
\end{tabular}




\section{Einstellungen zur Nutzung der Kern- energie und die Dringlichkeit des Ent- sorgungsproblems}

Eine wesentliche Frage der Studie bezieht sich darauf, inwieweit die Einstellungen zur Nutzung der Kernenergie die Haltung zur Frage der Entsorgung radioaktiver Abfälle beeinflusst. In der Ausgangsthese geht es darum, dass die Einstellungen zur Nutzung der Kernenergie insbesondere abhängig sind von der Kenntnis technischer Zusammenhänge bzw. der Kenntnis der Technik allgemein. In einer zweiten These wird vermutet, dass die Einstellung zum Entsorgungsproblem radioaktiver Abfälle in Deutschland abhängig ist von einer positiven Einstellung zur Nutzung der Kernenergie bzw. von positiven Bewertungen der Technik im Allgemeinen.

Die Tabelle 6 liefert einen Überblick zu den empirisch ermittelten Zusammenhängen. Sie werden anhand von Korrelationen für die eben vermuteten Beziehungen erläutert. lung zur Nutzung der Kernenergie verbunden sind.

Für die Einschätzung der eigenen Kenntnisse über moderne Technik und die Einschätzung der Dringlichkeit des Entsorgungsproblems in Deutschland gibt es demgegenüber keinen Zusammenhang. Beide Variablen sind unabhängig voneinander. Kenntnisse der modernen Technik haben damit keinen Einfluss darauf, ob das Entsorgungsproblem als dringlich oder nicht dringlich angesehen wird.

Die persönlichen Einstellungen zur Technik (vgl. zweite Spalte) und die Einstellungen zur Nutzung der Kernenergie besitzen einen relativ hohen Korrelationskoeffizienten von $.39^{* *}$. Dieses Ergebnis bestätigt weitgehend die Ausgangsthese. Wird jetzt jedoch wiederum die Dringlichkeit des Entsorgungsproblems betrachtet, so gibt es hier keinen nennenswerten Zusammenhang mit den persönlichen Einstellungen zur Technik.

Tab. 6: Korrelation der Kenntnisse und Einstellungen zur Technik mit den Einstellungen zur Nutzung der Kernenergie, der Dringlichkeit des Entsorgungsproblems und dem Grad der Information zum Thema „Entsorgung radioaktiver Abfälle“" (Korrelationskoeffizienten nach Pearson; $n=3.206$ )

\begin{tabular}{|l|c|c|c|c}
\hline & $\begin{array}{c}\text { Kenntnis der } \\
\text { Technik }\end{array}$ & $\begin{array}{c}\text { Einstellungen } \\
\text { zur Technik }\end{array}$ & $\begin{array}{c}\text { Einstellung zur } \\
\text { Nutzung der } \\
\text { Kernenergie }\end{array}$ & $\begin{array}{c}\text { Dringlichkeit } \\
\text { des Entsor- } \\
\text { gungsproblems }\end{array}$ \\
\hline Einstellungen zur Technik & $.45^{* *}$ & & & \\
\hline Einstellungen zur Nutzung der Kernenergie & $.26^{* *}$ & $.39^{* *}$ & & \\
\hline Dringlichkeit des Entsorgungsproblems & .03 & $.04^{*}$ & $-.20^{* *}$ & \\
\hline $\begin{array}{l}\text { Grad der Information über „Entsorgung } \\
\text { radioaktiver Abfälle“ }\end{array}$ & $.38^{* *}$ & $.20^{* *}$ & $.15^{* *}$ & $.04^{*}$ \\
\hline
\end{tabular}

** Signifikanz des Koeffizienten auf dem 1 \% Niveau; *Signifikanz des Koeffizienten auf dem 5 \% Niveau.

Aus der Tabelle 6 wird deutlich (vgl. erste Spalte), dass die eigenen Kenntnisse der modernen Technik und die persönlichen Einstellungen zur Technik besonders stark voneinander abhängig sind. ${ }^{9}$ Es wird hier ein Zusammenhang oder eine signifikante Korrelation von $.45^{* *}$ ermittelt. Die Einschätzung der eigenen Kenntnisse über moderne Technik besitzt aber auch eine Korrelation von .26** mit den Einstellungen zur Nutzung der Kernenergie. Das bedeutet, dass umfangreiche Kenntnisse technischer $\mathrm{Zu}$ sammenhänge mit einer eher positiven Einstel-
Diese Ergebnisse zeigen einen deutlichen Widerspruch. Kenntnisse und Einstellungen zur Technik sind zwar für die Einstellungen zur Nutzung der Kernenergie ausschlaggebend, jedoch nicht für die Beurteilung des Entsorgungsproblems.

Weiterhin zeigt die Tabelle 6 in der dritten Spalte, dass die Einstellungen zur Nutzung der Kernenergie einen negativen Korrelationskoeffizienten von $-.20 * *$ mit der Dringlichkeit des Entsorgungsproblems aufweisen. Dies bedeutet, dass eine positive Einstellung zur Nut- 
zung der Kernenergie eher mit einer nicht dringlichen Bewertung des Entsorgungsproblems einhergeht bzw. eine negative Einstellung zur Nutzung der Kernenergie mit einer sehr dringlichen Bewertung des Entsorgungsproblems. Dieses Ergebnis entspricht nicht der am Anfang des Abschnitts dargestellten zweiten These.

Schließlich zeigt die Tabelle 6 (vgl. letzte Zeile), dass diejenigen, die nach ihrer Einschätzung über gute Kenntnisse der Technik verfügen und diejenigen, die eine positive Einstellung zur Technik besitzen, sich auch sehr gut über das Thema „Entsorgung radioaktiver Abfälle“ informiert fühlen - die Korrelationskoeffizienten besitzen einen Wert von $.38 * *$ und $.20 * *$ und sind beide signifikant auf dem $1 \%$ Niveau.

Weiteres Ergebnis ist, dass eine positive Einstellung zur Nutzung der Kernenergie einhergeht mit einer weitgehend als ausreichend empfundenen Informationsbasis über das Thema der Entsorgung radioaktiver Abfälle. Der Korrelationskoeffizient beträgt $.15^{* *}$.

Der letzte in der Tabelle 6 aufgeführte $\mathrm{Zu}$ sammenhang bezieht sich auf die Dringlichkeit des Entsorgungsproblems und den Grad der Information über das Thema „Entsorgung radioaktiver Abfälle". Der Koeffizient ist gerade eben signifikant, jedoch kann man aufgrund der Höhe Korrelationskoeffizienten eher davon ausgehen, dass das Entsorgungsproblem als unabhängig vom Informationsgrad bewertet wird.

\section{Zusammenfassung}

Hinsichtlich der Glaubwürdigkeit der Informationen verschiedener Institutionen zur Nutzung der Kernenergie in Deutschland agiert der AkEnd als Expertenkommission in einem Themenfeld, indem auf der einen Seite den Informationen über die Nutzung der Kernenergie eine recht hohe Glaubwürdigkeit entgegengebracht wird (wenn sie von Umweltforschungsinstituten stammen), andererseits jedoch auch eine hohe Unglaubwürdigkeit zu konstatieren ist (von der Atomwirtschaft). Auf den AkEnd kommt hier eine hohe Integrationsaufgabe $\mathrm{zu}$, wenn einerseits Umweltforschungsinstitute und andererseits die Atomwirtschaft an der Standortsuche für ein Endlager beteiligt werden.

Wenn ein Endlager für radioaktive Abfälle in der Region entstünde und ob die Befragten dies akzeptierten, wird von einer deutlichen Mehrheit von 80,6 \% ausgeschlossen. Ein ähnliches Phänomen („,not-in-my-backyard“) lässt sich für ein gemeinsames Endlager für radioaktive Abfälle der Europäischen Gemeinschaft feststellen, das in Deutschland angesiedelt sein könnte. 38,1\% sind dafür, 41,7\% dagegen und $20,2 \%$ der Befragten sind unentschieden.

Einen deutlichen Widerspruch zeigen die Ergebnisse der Korrelationsanalyse. Kenntnisse und Einstellungen zur Technik sind zwar für die Einstellung zur Nutzung der Kernenergie ausschlaggebend, jedoch nicht für die Beurteilung des Entsorgungsproblems von radioaktiven Abfällen.

Weiterhin konnte gezeigt werden, dass die Einstellung zur Nutzung der Kernenergie einen negativen Koeffizienten mit der Dringlichkeit des Entsorgungsproblems aufweist. Dies bedeutet, dass eine positive Einstellung zur Nutzung der Kernenergie eher mit einer nicht dringlichen Bewertung des Entsorgungsproblems einhergeht bzw. eine negative Einstellung zur Nutzung der Kernenergie mit einer sehr dringlichen Bewertung des Entsorgungsproblems.

Diejenigen Befragten, die nach ihren Einschätzungen über gute Kenntnisse der Technik verfügen, und diejenigen, die eine positive Einstellung zur Technik besitzen, fühlen sich auch sehr gut über das Thema „Entsorgung radioaktiver Abfälle“ informiert.

Der AkEnd legt am Ende des Jahres 2002 sein Gutachten dem Bundesumweltministerium (BMU) vor und wird bis dahin von ITAS evaluiert. Das ITAS-Projekt wird seinen Abschlussbericht bis Ende diesen Jahres erstellen.

\section{Anmerkungen}

1) http://www.AKEND.de

2) Wertorientierungen sind nach dem IngelhartIndex empirisch ermittelt.

3) BIK-Systematik: Indikator zur Beschreibung von Siedlungs- und Verflechtungsstrukturen in Deutschland, der auf den Boustedt-Regionen basiert. Für die Entwicklung der Systematik wurden bundesweite Pendlerstromanalysen verwendet. Entwickelt wurde die Systematik vom Institut BIK Aschpurwis+Behrens.

4) Das Minimum der Skala beträgt 1 für ,äußerst unwichtig“ und das Maximum besitzt den Wert 7 für ,äußerst wichtig“ - dazwischen liegende 
Kategorien sind jeweils nicht bezeichnet.. Die Berechnung des Mittelwertes basiert auf diesem Skalenbereich.

5) Das Minimum der Skala beträgt 1 für ,sehr wichtig“ und das Maximum besitzt den Wert 4 für ,ganz unwichtig“ - dazwischen liegende Kategorien sind jeweils bezeichnet. Die Berechnung des Mittelwertes basiert auf diesem Skalenbereich.

6) Das Minimum der Skala beträgt 1 für ,,völlig glaubwürdig“ und das Maximum besitzt den Wert 5 für ,völlig unglaubwürdig“ - dazwischen liegende Kategorien sind jeweils bezeichnet. Die Berechnung des Mittelwertes basiert auf diesem Skalenbereich.

7) Das Minimum der Skala beträgt 1 für ,eine sehr geringe Bedeutung“ und das Maximum besitzt den Wert 7 für ,eine sehr hohe Bedeutung“ dazwischen liegende Kategorien sind jeweils nicht bezeichnet. Die Berechnung des Mittelwertes basiert auf diesem Skalenbereich.

8) Das Minimum der Skala beträgt 0 für „sehr unwahrscheinlich“ und das Maximum besitzt den Wert 1 für ,sehr wahrscheinlich“ - dazwischen liegende Kategorien sind jeweils bezeichnet. Die Berechnung des Mittelwertes basiert auf diesem Skalenbereich.

9) Die erste Frage lautet: Wie schätzen Sie Ihre eigenen Kenntnisse über die moderne Technik und technische Zusammenhänge ein?“. Die zweite Frage lautet: ,Wenn Sie an Ihre persönliche Einstellung zur Technik denken: Wo würden Sie sich selbst auf dieser Skala einstufen?“”.

\section{Kontakt}

Dr. Martin Stolle

Forschungszentrum Karlsruhe GmbH

Institut für Technikfolgenabschätzung und Systemanalyse (ITAS)

Postfach 36 40, 76021 Karlsruhe

Tel.: +49 (0) 7247 / 82 - 6795

Fax: +49 (0) 7247 / 82 - 6045

E-Mail: martin.stolle@itas.fzk.de

Internet: http://www.itas.fzk.de

\section{《〉}

